\title{
FACTORES DETERMINANTES EN LA TASA DE RENDIMIENTO EN UNA EMPRESA DEL SECTOR COSMÉTICO
}

Raquel Caro-Carretero y Susana Ortiz-Marcos

UNIVERSIDAD PONTIFICIA COMILLAS. Escuela Técnica Superior de Ingeniería (ICAI). Dpto. de Organización Industrial. C/Alberto Aguilera 25, 28015 - Madrid. Tfno: +34 915422800.

rcaro@upcomillas.es

Recibido: 15/oct/2013 - Aceptado: 26/dic/2013 - DOI: http://dx.doi.org/10.6036/MN6983

\section{EXPLANATORY FACTORS FOR THE PERFORMANCE RATE IN A COSMETIC COMPANY SECTOR}

\begin{abstract}
:
Nowadays, most companies are managing large amounts of information and they often lack the ability to use them as a competitive advantage in their decision-making process. This research applies statistical analysis to the information provided by a company in the cosmetics industry, not only in a descriptive but in an explanatory and predictive function. Data collection corresponds to two production units of a factory, hairdressing and coloring, with different packaging lines in the conditioning phase. Production lines performance is paramount to take advantage of them, as well as improving efficiency and productivity. A systematic way to monitor the use of productive resources is the indicator Overall Equipment Effectiveness (OEE). The advantage of OEE is that it measures in a single indicator all parameters of industrial production: availability, performance and quality. The contribution of this study is to identify factors that cause the deviation of these both rates respect the target previously set by corporate management. A logistic regression model has been formulated. As a practical contribution of this paper, a series of recommendations within the program of continuous improvement of the plant is proposed, promoting the effort to involve employees in this "learning process". The main finding of this study is the explanatory power of production time loss, rather than time inefficiencies in the production line management. The most significant time loss is due to formula or reference or format changes. The combined effect of microstoppages and change of workshift does significantly contribute to the explanatory power of the model.

Keywords: Overall Equipment Effectiveness, Continuous Improvement, Lean Manufacturing, logistic regression models

\section{RESUMEN:}

Hoy en día, la mayoría de las empresas se enfrentan a grandes cantidades de información y datos, careciendo, en muchos casos, de la capacidad de aprovecharlos como ventaja competitiva en su toma de decisiones. Este trabajo de investigación aplica el análisis estadístico a la información proporcionada por una empresa del sector cosmético, no solo de una manera descriptiva, sino explicativa y predictiva basándose en modelos de análisis multivariante. La recogida de datos corresponde a dos unidades de producción de una fábrica, peluquería y coloración, con distintas líneas de envasado dentro de la fase de acondicionamiento. El rendimiento de las líneas de producción es vital para un mejor aprovechamiento de las mismas, así como una mejor eficacia y productividad. Una forma sistemática de controlar el uso de los recursos productivos es el indicador de Efectividad Global de los Equipos (OEE). La ventaja del OEE es que mide todos los parámetros de la producción industrial: disponibilidad, productividad y calidad en un solo indicador. De acuerdo a la tasa que utiliza dicha fábrica para el cálculo de la disponibilidad y la productividad de las líneas (Tasa de Rendimiento Sintético, TRS) se propone, como aportación del trabajo, mejorar la métrica del OEE. Para ello, se plantea como objetivo general identificar los posibles factores que provocan que la TRS real de las líneas de producción se aleje respecto de la TRS objetivo fijada previamente por la dirección de la empresa. Se formula para ello un modelo de regresión logística. Una vez detectados, y como contribución práctica de este trabajo, se propone la puesta en marcha de una serie de recomendaciones dentro del programa de Mejora Continua de la fábrica, promoviendo el esfuerzo por involucrar a los empleados en este "proceso de aprendizaje". Las conclusiones de este estudio son que las variables explicativas en la predicción del rendimiento por línea más importantes se asocian al tiempo de pérdida, más que al tiempo de ocupación de las líneas, siendo la más significativa el tiempo de pérdida por cambio de fórmula, de referencia o de formato. El efecto combinado del tiempo de pérdida por microparos con el turno laboral sí contribuye al poder explicativo de la diferencia entre las TRS.

Palabras clave: Efectividad Global del Equipo (OEE), Mejora Continua, Lean Manufacturing, modelos de regresión logística
\end{abstract}




\section{INTRODUCCION}

En una época como la actual, tan compleja desde el punto de vista económico y de competitividad, cada vez resulta más difícil mantenerse satisfactoriamente en el mercado. Especialmente en aquellas empresas cuya actividad fundamental son los procesos de transformación, la mejora de la competitividad se ha convertido en objetivo prioritario, resultando los programas de estrategia de Mejora Continua esenciales en la planificación de sus procesos productivos [1]. La Mejora Continua (kaizen) significa una mejora incremental dinámica basada en el conocimiento. Este término fue introducido por primera vez por Masaaki Imai [2] y lo define como: "Mejoramiento y, aún más, mejoramiento continuo que involucra a todos, gerente y trabajadores por igual". Es decir, Mejora Continua representa un complejo proceso en evolución de "learning-by-doing" (aprendiendo con la práctica) y el fomento de la capacidad organizativa [3-4]. Aunque las empresas son conscientes de lo que supone la Mejora Continua para su supervivencia, les queda un largo camino por recorrer. Autores como Suárez-Barraza et al [5-6], demuestran empíricamente en dos casos de estudio que existe una brecha entre los esquemas teóricos del kaizen y la realidad práctica y que aunque su significado puede parecer sencillo y de sentido común, la realidad muestra que su aplicación es complicada si no hay un cambio de pensamiento y organización radical que se mantenga a lo largo del tiempo. En este sentido resulta importante la formación en herramientas clásicas de mejora, siendo el análisis estadístico, entre otras, una de ellas [7-10].

Además, en este contexto de mejora de la competitividad, la filosofía del Lean Manufacturing ayuda a afrontar las dificultades y superarlas desde una posición más favorable [11]. Aplicado a un sistema productivo Lean Manufacturing significa "esbelto" o "ajustado". Hay muchas definiciones propuestas por diversos autores. Por ejemplo, Womack y Jones [12-13] describen el pensamiento Lean como la búsqueda de la perfección como antídoto contra el muda o "desperdicio", entendiendo como tal todo aquello que no añade valor al producto o que no es absolutamente esencial para fabricarlo e introduciendo la importancia del capital humano como pieza fundamental. Dichos autores proponen en definitiva un cambio cultural radical. Este cambio consiste en analizar y medir la eficiencia y productividad de todos los procesos en términos de "valor añadido" y "despilfarro o muda". La eliminación de estos "desperdicios" disminuirá el coste de producción, acortará el lead time (tiempo que tarda una unidad en atravesar todo el proceso de producción: desde la recepción del pedido hasta la entrega del producto al cliente), y favorecerá un flujo continuo en la fábrica. Estos "desperdicios" engloban sobreproducción, movimientos innecesarios, inventario, transporte, defectos y sobrecarga y tiempos de espera. En este concepto están incluidas todas las fuentes de ineficiencia, estén o no programadas, ya que la única manera es partir de la base de identificar todo para trabajar después sobre lo que es susceptible de mejora.

Esta filosofía o metodología de producción ha aportado a un buen número de empresas - que se mantienen airosas en la difícil situación actual-una mayor rapidez y flexibilidad en su respuesta ante los cambios del mercado. Lean aporta una nueva forma de medir la eficiencia y la productividad en los sistemas productivos. Aspectos éstos fundamentales ante un consumidor que continuamente exige cambios tanto en diseños como en prestaciones del producto. Algunos autores han demostrado cómo la aplicación de dicha metodología es un arma muy valiosa para eliminar "desperdicios" y mejorar la tasa de rendimiento [14-15].

Y entre las herramientas propuestas para implementación de Lean aparecen las prácticas avanzadas de producción de Mantenimiento Productivo Total (Total Productive Maintenance, TPM en adelante) para cuantificar el rendimiento global de diferentes plantas [16]. El TPM es entendido como un conjunto de múltiples acciones que persigue eliminar las pérdidas por tiempos de parada de las máquinas. Son los mismos operarios de producción los que realizan las tareas de TPM de forma autónoma, haciéndose cargo de las técnicas necesarias y proponiendo mejoras en las máquinas que afecten a nuevos diseños de línea. Una vez aquí, resulta de gran importancia definir un sistema de indicadores accesible y fiable para capturar, medir, analizar y evaluar los resultados y desviaciones respecto al objetivo de manera metódica y fiable. En Lean, el indicador numérico natural para el TPM es la tasa de Efectividad Global de los Equipos (Overall Equipment Effectiveness, OEE en adelante).

Este indicador compara el número de piezas que se podrían haber producido (si todo hubiera ido según lo previsto) y las unidades sin defectos que realmente se han producido. No trata de justificar las razones que explican la desviación, sino identificar las pérdidas para poder erradicar las causas que las originan. También se puede definir como la relación entre 
el tiempo neto que el equipo tarda en hacer una unidad "buena" y el número total de horas que se espera que funcione el equipo durante un período de tiempo dado.

La ventaja del OEE es que mide, en un único indicador, todos los parámetros fundamentales en la producción industrial en tres tasas: de disponibilidad, de productividad y de calidad, constituyendo una de las claves del Lean. De manera que es posible saber si lo que falta hasta el $100 \%$ se ha perdido por disponibilidad (la maquinaria estuvo cierto tiempo parada), eficiencia o productividad (la maquinaria estuvo funcionando a menos de su capacidad total) o calidad (se han producido unidades defectuosas). De ahí que el OEE se ha convertido en un estándar internacional reconocido por las principales industrias alrededor del mundo. Así, la mejora de la efectividad con la que trabajan los equipos y las instalaciones permite el incremento de la efectividad de todo el sistema productivo. El valor numérico de la efectividad global es un porcentaje que se haya antes de introducir mejoras para conocer el punto de partida del equipo cuya efectividad se quiere incrementar. Autores como Mansour et al. [17] han demostrado empíricamente (en el caso particular de perforación de pozos) como el OEE ayuda a identificar exactamente los problemas para poder corregirlos.

El OEE es el producto de estas tres tasas:

\section{OEE $(\%)=$ DISPONIBILIDAD*PRODUCTIVIDAD*CALIDAD}

En la Fig. 1 se muestra la relación entre los distintos componentes del OEE.

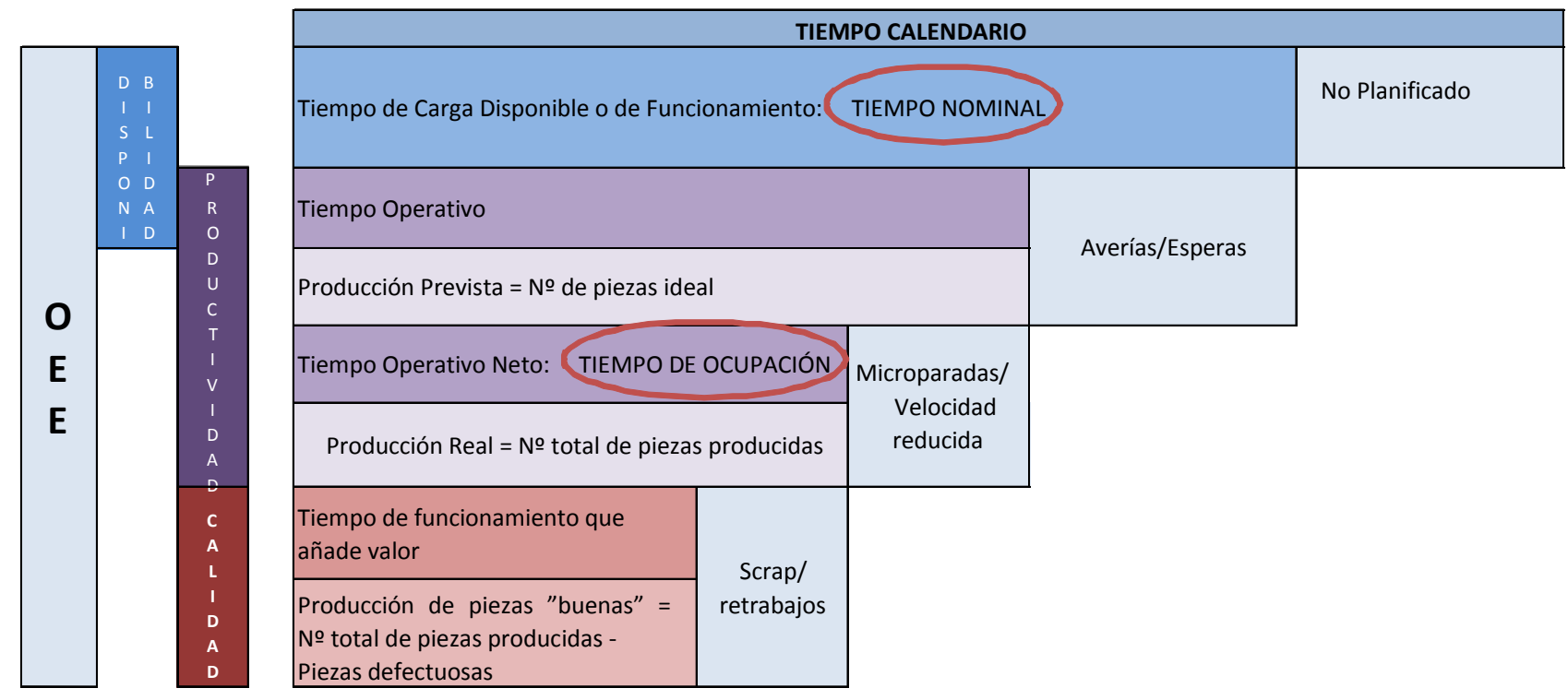

Fig. 1. Esquema de los componentes del indicador OEE

De acuerdo a este esquema tenemos el cálculo de las distintas tasas como sigue a continuación:

$$
\begin{gathered}
\text { DISPONIIBILIDAD }(\%)=\frac{\text { Tiempo Operativo }}{\text { Tiempo de carga disponible }} \\
\text { PRODUCTIVIDAD }(\%)=\frac{\text { Tiempo Operativo Neto }}{\text { Tiempo Operativo }}=\frac{\text { Producción Real }}{\text { Producción Prevista }} \\
\text { CALIDAD }(\%)=\frac{\text { Tiempo Operativo que añade valor }}{\text { Tiempo Operativo Neto }}=\frac{\text { Piezas "buenas" }}{\text { Producción Real }}
\end{gathered}
$$


El primer gran bloque de pérdidas afecta a la tasa de Disponibilidad (fracción de tiempo que el equipo está operando realmente) y refleja las pérdidas por averías y paradas generadas por esperas. Para hacer frente a las mismas es fundamental contar con algún sistema o al menos una planilla de cálculo sencilla que permita obtener esta información con la frecuencia deseada, a partir de la cual se podrán recoger los datos, estratificarlos, elaborar diagramas causa-efecto y el tan reconocido diagrama de Pareto, así como utilizar otras herramientas más sofisticadas como el histograma o los gráficos de control. El objetivo principal es identificar los problemas que generan las averías y paradas para trabajar sobre sus causas y así poder eliminarlas sistemáticamente.

El segundo gran bloque de pérdidas afecta a la Productividad o Eficiencia de operación, la cual mide el nivel de funcionamiento del equipo teniendo en cuenta las pérdidas por tiempos muertos, paradas menores y pérdidas por una velocidad más baja que la de diseño. El problema principal suele darse por cambios y puestas en marcha. Esto puede abordarse de la misma manera que la disponibilidad y con técnicas SMED (Single Minute Exchange of Die-tiempo de preparación de un solo dígito o tiempo de cambio inferior a 1 minuto). SMED propone convertir las preparaciones internas de las máquinas en actividades externas, es decir, poder realizar dichas actividades con la máquina en marcha.

Para el tercer bloque de pérdidas, la tasa de Calidad mide la fracción de la producción obtenida que cumple los estándares de calidad, reflejando aquella parte del tiempo empleada en la fabricación de piezas con defectos. Se pueden volver a emplear las técnicas del primer bloque añadiendo algunas herramientas estadísticas como los estudios de capacidad de proceso basados en la dispersión (Cp o CpK) o Seis Sigma.

El trabajo que aquí se presenta surge del interés en profundizar en el campo de gestión de la calidad y rendimiento de los procesos de fabricación como estrategia corporativa en una fábrica del sector cosmético con implantación Lean y centrándolo, a petición de la misma, en un tipo de "desperdicios": tiempos no productivos que provocan que no haya un flujo continuo generando tiempos de parada y la consiguiente bajada en la tasa de rendimiento. En este sentido, el indicador utilizado por la dirección de esta empresa es la Tasa de Rendimiento Sintético (Taux de Rendement Synthétique acorde con la norma NFE 60-182, TRS en adelante). Para su cálculo se tienen en cuenta las tasas de Productividad y la de Disponibilidad que forman parte del indicador OEE.

Los estudios descriptivos sobre la OEE, basados en su métrica, se utilizan para obtener conocimiento y comprensión del problema de investigación [17-19], y no suelen aplicar las consideraciones del análisis estadístico explicativo y/o predictivo. De esta forma, se publican unos resultados muy generales del comportamiento de la OEE. Teniendo en cuenta esta necesidad, el trabajo con un enfoque cuantitativo que se presenta busca la aplicación del análisis estadístico a la información proporcionada por la entidad. Por tanto, el objetivo, en este contexto, es la identificación de los factores explicativos estadísticamente del primer y segundo factores de pérdidas del indicador OEE que afectan al rendimiento de operaciones en la fábrica objeto de estudio. Un vez detectados, como un primer paso en el camino a recorrer en un entorno Lean, se propone la puesta en marcha de medidas que hagan de las mejoras un estándar en esta fábrica y, con la posibilidad de su extensión a otras del grupo.

En concreto, se pretende utilizar la Estadística en el marco de trabajo específico de la organización de la producción sobre la base de un conjunto de estrategias que se centran en la mejora del OEE mediante un modelo explicativo de regresión logística, complementando así las herramientas clásicas típicamente descriptivas y exploratorias. La integración del "pensamiento y métodos estadísticos" en los procesos empresariales (con el apoyo e implicación de la alta dirección), puede crear la infraestructura y las habilidades que permitan a la organización utilizar la Estadística como ventaja competitiva en la toma de decisiones [20].

El artículo introduce el esquema del proceso de producción de la entidad, así como el indicador de rendimiento que utiliza para controlar la velocidad de envasado. A continuación se detalla la muestra y la hipótesis de investigación junto con la metodología empleada. Posteriormente se presentan los resultados y la discusión de los mismos a modo de conclusión. 


\section{DESCRIPCIÓN DEL PROCESO}

La Fig. 2. muestra un esquema del proceso de fabricación en las dos unidades de producción (UP en adelante) analizadas: Peluquería (UP1) y Coloración (UP2). Cada UP se divide en dos procesos: Fabricación y Acondicionamiento. En el proceso de Fabricación se fabrica el jugo y en el proceso de Acondicionamiento se introduce el jugo en el envase específico. Se presenta un único diagrama de flujo porque es igual para ambas unidades de producción (UP1 y UP2), aunque tanto las materias primas como los equipos son diferentes para cada una de las UP. Así, en la UP1, correspondiente a Peluquería, los productos terminados son champús, cremas, acondicionadores, mascarillas, geles..., mientras que en la UP2, correspondiente a Coloración, los productos terminados son tintes.

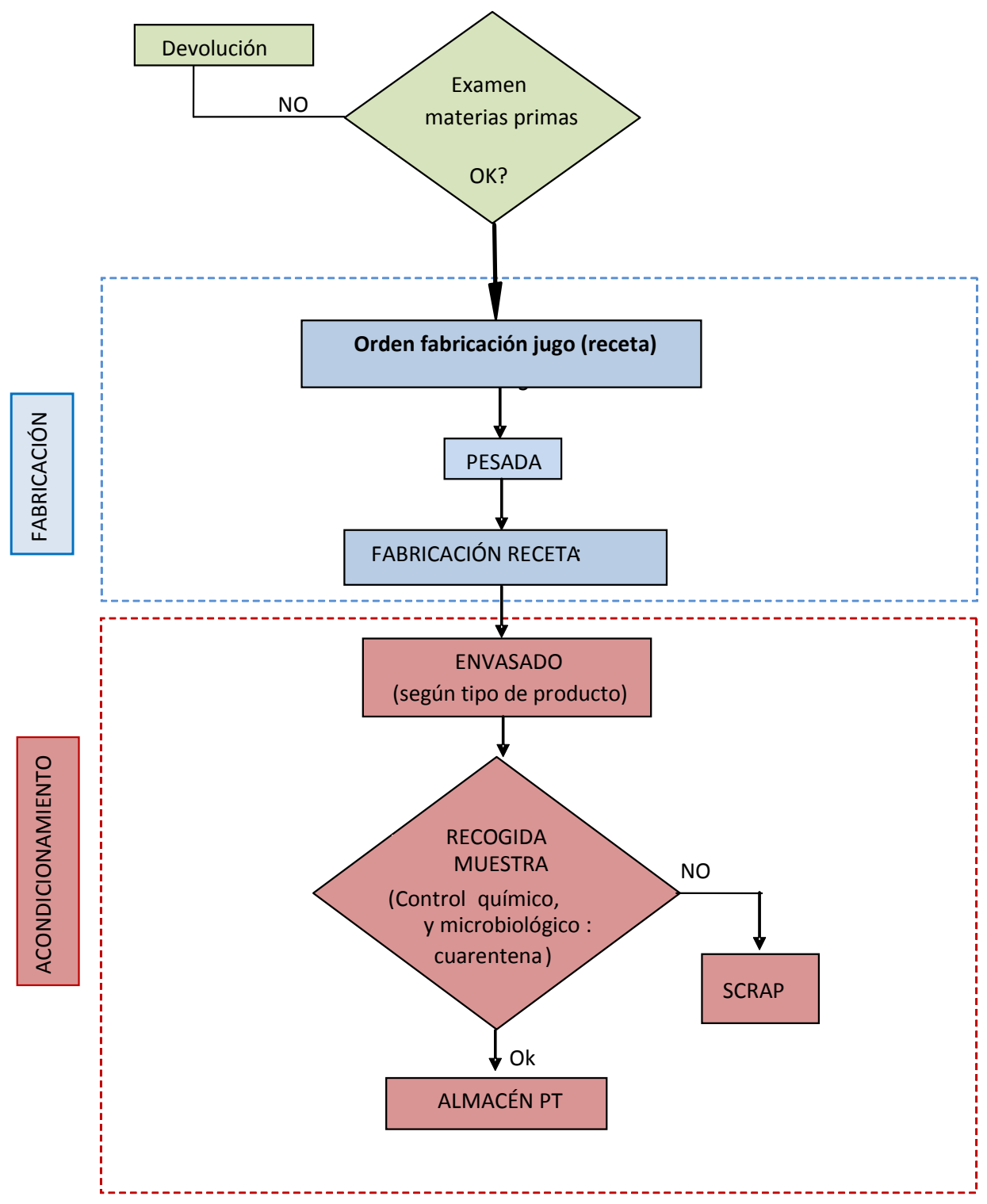

Fig. 2: El proceso de fabricación 


\subsection{PROCESO DE FABRICACIÓN}

El proceso de fabricación se divide a su vez en Pesadas y Fabricación.

- Pesadas: Es la zona donde se pesa cada una de las materias primas que forman una orden de fabricación (Jugo). Dichas materias primas han sufrido previamente un examen riguroso de calidad que es el que autoriza su posterior utilización.

- Fabricación: A través de una receta y siguiendo el orden y método marcados por la dirección se introducen las materias primas, previamente pesadas. A continuación, los equipos de fabricación tratan los distintos componentes para producir el jugo.

Una vez completado el proceso de fabricación se transporta el jugo, mediante tuberías, a la línea de producción.

Tanto la Pesada como la Fabricación son controladas por un software donde en todo momento el operador recibe y visualiza la información necesaria para poder llevar a cabo cada una de las tareas.

\subsection{EL PROCESO DE ACONDICIONAMIENTO}

Acabada la orden de Fabricación se iniciará una Orden de Acondicionamiento. El software anterior permite al operador verificar de cuántas unidades es la orden de acondicionamiento a realizar, si el jugo fabricado está en perfectas condiciones según los estándares de Calidad y si tiene todos los componentes que forman el producto y en las cantidades necesarias.

Cada caja y palé producidos van identificados con una etiqueta que permitirá asegurar la trazabilidad del producto. Una vez acabado este proceso y durante el mismo, una serie de muestras recogidas para el control químico, físico y microbiológico aseguran la calidad del producto durante y después del envasado. Si después de estar en cuarentena las muestras no superan los requisitos de calidad, se desechan. Los cambios de envases se realizan de forma manual en las líneas.

\subsection{CONSIDERACIONES PREVIAS EN LA TASA DE RENDIMIENTO}

A continuación se definen las variables utilizadas en el indicador TRS que utiliza la empresa objeto de estudio y que nos permitirá estudiar la disponibilidad y el rendimiento de cada una de las líneas.

El tiempo nominal, que puede variar considerablemente según las condiciones en que opera la máquina o línea, se define como el tiempo teórico que indica la velocidad o eficiencia de envasado declarada en la especificación (Normativa DIN 8743). De tal manera que si no se cumple dicha velocidad se registra como tiempo de pérdida (breakdowns) en esperas o averías (permitido un 2\% del tiempo nominal) o microparos (permitido un 5\% del tiempo nominal). No se dispone de cronometrajes. Este tiempo nominal, que depende de la cadencia de cada línea, informa de cuánto se debería tardar realmente.

El tiempo de ocupación es el tiempo real empleado por el operador en la línea para terminar todas las unidades planificadas. En él se incluyen los tiempos perdidos en averías, esperas, limpieza, mantenimiento, cambios de formato, jugo o referencia y otros microparos surgidos en la línea.

La relación entre ambas variables es la TRS real, indicador que siguen los operadores de las líneas de producción. La dirección de la fábrica fija una TRS objetivo que refleja el best rank entre todas las fábricas de la entidad. Cada UP debe trabajar para conseguir individualmente dicho objetivo. 
De acuerdo al esquema de componentes del OEE reflejado en la Fig.1, la TRS se podría definir como producto de las dos primeras razones porcentuales tal que

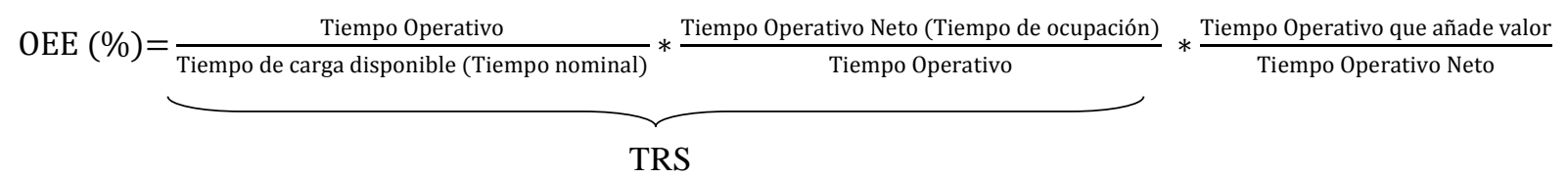

\section{MATERIALES Y METODOS}

\subsection{PROVISIÓN DE DATOS, SELECCIÓN Y ADAPTACIÓN DE LA INFORMACIÓN. LA MUESTRA Y LA HIPÓTESIS DE INVESTIGACIÓN}

Con el apoyo y la implicación de la dirección de la entidad, se nos proporciona una recogida de datos interna que corresponde a marzo de 2013 de las dos mencionadas unidades de producción: Peluquería y Coloración, con 20 y 19 líneas respectivamente correspondientes a la fase de acondicionamiento. Para cada línea se confeccionó un registro diario con información de tiempos de ocupación, tiempos de pérdida por averías, microparos, esperas o cambios de formato, referencia o jugo (fórmula), así como información porcentual de las tasas de rendimiento objetivo y real. Adicionalmente se tiene constancia para cada registro del turno laboral (mañana, tarde o noche) en que se produce. Cada línea, de acuerdo con la TSR real respecto de la TRS objetivo, ha sido clasificada en uno de los 2 grupos siguientes: "línea se aleja por debajo de la tasa de rendimiento objetivo" y "línea se aleja por encima de la tasa de rendimiento objetivo". Sobre este conjunto de variables, definidas según la Tabla 1 se aplican las técnicas de análisis multivariable [21].

\begin{tabular}{ll}
\hline Variables & Definición \\
\hline Tiempo de ocupación & $\begin{array}{l}\text { Tiempo real empleado por el operador en la línea para terminar } \\
\text { todas las unidades planificadas }\end{array}$ \\
\hline $\begin{array}{l}\text { Tiempos de pérdida por cambios de formato jugo } \\
\text { (fórmula) o referencia }\end{array}$ & Aquellas paradas planificadas (de 45', 20’ y 10’, respectivamente) \\
\hline Tiempo por mantenimiento preventivo & Aquellas paradas planificadas mecánicas \\
\hline Tiempo de limpieza & Aquellas paradas planificadas para limpieza \\
\hline Tiempo de pérdida por esperas & $\begin{array}{l}\text { Tiempos no planificados de espera de jugo, artículos, carretilleros, } \\
\text { mecánico, etc. }\end{array}$ \\
\hline Tiempo de pérdida por averías & $\begin{array}{l}\text { Paradas no planificadas por alguna avería en alguna máquina que } \\
\text { forma parte de la línea }\end{array}$ \\
\hline Tiempo de pérdida por microparos & Aquellas paradas no planificadas distintas de las anteriores \\
\hline Tasa de rendimiento & Ratio entre tiempo nominal y tiempo de ocupación \\
\hline Turno laboral & M mañana, T = tarde, N = noche \\
\hline Unidades de producción & $1=$ UP1, 2=UP2 \\
\hline Línea se aleja de la tasa de rendimiento objetivo & $=0$ por debajo, = 1 por encima \\
\hline
\end{tabular}

Tabla 1: Definición de variables que se van a emplear en los análisis multivariantes

La fábrica objeto de esta investigación decide implantar los comúnmente llamados Programas de Mejora Continua, definidos como "una combinación de tareas, orientaciones y actuaciones necesarias para poder incluir los conceptos y el uso de la mejora continua de la calidad, en el desarrollo de la actividad directiva" [7]. En el marco de estos programas una de las actividades que se suele considerar e introducir es la resolución de problemas mediante Equipos de mejora. No obstante, siendo los "métodos" a la hora de solucionar los problemas tan racionales y tan basados en el sentido común, su utilización es difícil de implantar y mantener a lo largo del tiempo en las empresas por las condiciones del entorno en que se pretenda aplicar el método. Y uno de los condicionantes del entorno más importantes a la hora de aplicar los programas de mejora trabajando en equipo en las empresas es el elemento humano. Son las personas las que 
tienen que aplicarlos. Estos programas son en realidad "programas de aprendizaje” basándose en la experiencia. En este sentido la entidad objeto de estudio implanta talleres Kaizen de periodos cortos de tiempo cuando las desviaciones a la TRS objetivo se deben a pérdidas conocidas y talleres de mejora continua de periodos de tiempo de al menos 3 meses donde se implica a más personal y se estudian aquellas pérdidas desconocidas. Resulta por lo tanto esencial conocer los factores que facilitan y que inhiben dicho aprendizaje.

Por todo lo anterior, y para cumplir con el objetivo de este trabajo se plantea estudiar la siguiente hipótesis de investigación: La identificación de los factores explicativos que provocan las diferencias significativas estadísticamente entre la tasa de rendimiento real y la tasa de rendimiento objetivo por línea de dos unidades de producción mejorará los "programas de aprendizaje". Por tanto, un primer paso sería detectar dentro de las dos unidades de producción objeto de estudio dónde y por qué se producen los "mudas" o tiempos no productivos que provocan una bajada de la tasa de rendimiento. Posteriormente se plantean recomendaciones en torno a la TRS como estrategia de mejora del indicador OEE.

\subsection{TÉCNICAS DE PROCESAMIENTO Y ANÁLISIS DE DATOS}

Se ha llevado a cabo un estudio cuantitativo para establecer si existe algún modelo explicativo (y/o predictivo) de la variable "la línea se aleja de la TRS objetivo".

\begin{tabular}{|c|c|c|c|}
\hline $\begin{array}{l}\text { Metodología } \\
\text { empleada }\end{array}$ & Objetivos & $\begin{array}{l}\text { Variable } \\
\text { dependiente } \\
\text { "respuesta" }\end{array}$ & $\begin{array}{l}\text { Variables independientes o } \\
\text { "predictoras" }\end{array}$ \\
\hline $\begin{array}{l}\text { El análisis } \\
\text { exploratorio } \\
\text { descriptivo }\end{array}$ & $\begin{array}{l}\text { Describir la muestra y examinar los datos } \\
\text { previamente a la aplicación de las técnicas } \\
\text { estadísticas multivariables }\end{array}$ & ---------------- & ------------- \\
\hline $\begin{array}{l}\text { Contraste T de } \\
\text { medias y } \\
\text { proporciones }\end{array}$ & $\begin{array}{lccc}\text { Examinar } & \text { si } & \text { existen } & \text { diferencias } \\
\text { estadísticamente } & \text { significativas } & & \end{array}$ & --------------- & $\begin{array}{ll}\text { - } & \text { Proporción de líneas que se } \\
& \text { alejan de la TRS objetivo } \\
\text { - } & \text { TRS media por UP } \\
\text { - } & \text { Tiempo medio de pérdida } \\
& \text { por UP } \\
\text { - } & \text { Tiempo medio de cambio por UP } \\
\text { - } & \text { Tiempo medio de averías por UP } \\
\text { - } & \text { Tiempo medio de microparos por } \\
& \text { UP }\end{array}$ \\
\hline $\begin{array}{l}\text { Análisis } \\
\text { Discriminante }\end{array}$ & $\begin{array}{l}\text { Identificar las variables que contribuyen en } \\
\text { mayor grado a discriminar } \\
\text { significativamente las líneas en las dos } \\
\text { unidades de producción. }\end{array}$ & $\begin{array}{l}\text { Unidades } \\
\text { producción }\end{array}$ & $\begin{array}{ll}\text { - } & \text { Tiempo de pérdida } \\
\text { - } & \text { Tiempo de ocupación } \\
\text { - } & \text { Turno laboral } \\
\text { - } & \text { Tiempo por limpieza } \\
\text { - } & \text { Tiempo por mantenimiento } \\
\end{array}$ \\
\hline $\begin{array}{l}\text { Análisis de } \\
\text { Regresión lineal } \\
\text { múltiple }\end{array}$ & $\begin{array}{l}\text { Medir el grado de asociación entre las } \\
\text { variables consideradas }\end{array}$ & $\begin{array}{l}\text { Diferencia entre la } \\
\text { TRS real y la } \\
\text { objetivo }\end{array}$ & $\begin{array}{l}\text { - Tiempo de averías } \\
\text { - Tiempo de esperas } \\
\text { - Tiempo de microparos } \\
\text { - Tiempo de cambios de formato, } \\
\text { jugo o referencia }\end{array}$ \\
\hline $\begin{array}{l}\text { Análisis de } \\
\text { Regresión logística }\end{array}$ & $\begin{array}{l}\text { Identificación de los factores explicativos de } \\
\text { la diferencia entre la TRS objetivo y real }\end{array}$ & $\begin{array}{l}\text { Línea se aleja de la } \\
\text { TRS objetivo }\end{array}$ & $\begin{array}{l}\text { - Turno laboral } \\
\text { - Tiempo de ocupación } \\
\text { - Tiempo de pérdida por avería, por } \\
\text { microparos, por esperas o por } \\
\text { cambio de formato, jugo o } \\
\text { referencia }\end{array}$ \\
\hline
\end{tabular}

Tabla 2: Resumen explicativo de objetivos y variables a considerar en cada técnica estadística empleada 
Dado que las dos unidades de producción se diferencian estadísticamente de manera significativa en este indicador de rendimiento, el propósito ha sido identificar qué variables permiten diferenciar a los grupos de líneas que se alejan por debajo o por encima de la TRS objetivo en cada una de las unidades de producción. Se formula para ello un análisis de regresión logística binaria. Con este modelo de regresión se pretende constatar el efecto de las características de cada una de las líneas consideradas en la probabilidad de que la TRS real se aleje respecto de la TRS objetivo por línea. En este sentido, adicionalmente se utilizan otras herramientas estadísticas para complementar el análisis obtenido y profundizar en los resultados. Con ello, se ha conseguido un entendimiento básico de los datos y de las relaciones existentes entre las variables analizadas.

Cada técnica estadística utilizada tiene sus propios fines como muestra de manera resumida la Tabla 2 . Se ha identificado las variables "respuesta" e independientes a utilizar en cada técnica estadística.

El análisis de los datos y las comprobaciones de los supuestos subyacentes en las técnicas multivariantes se han realizado a través del software estadístico IBM SPSS Statistics 20.

\section{RESULTADOS}

\subsection{ANÁLISIS EXPLORATORIO}

De las 39 líneas que forman el total de la muestra, 22 están por debajo de la tasa objetivo, siendo 12 (54,5\%) de ellas correspondientes a la UP1. Dentro de la UP1 un 60\% de las líneas presenta una tasa de rendimiento real inferior a la tasa de rendimiento objetivo. En la UP2 este porcentaje asciende a 52,6\% según muestra la Fig. 2.

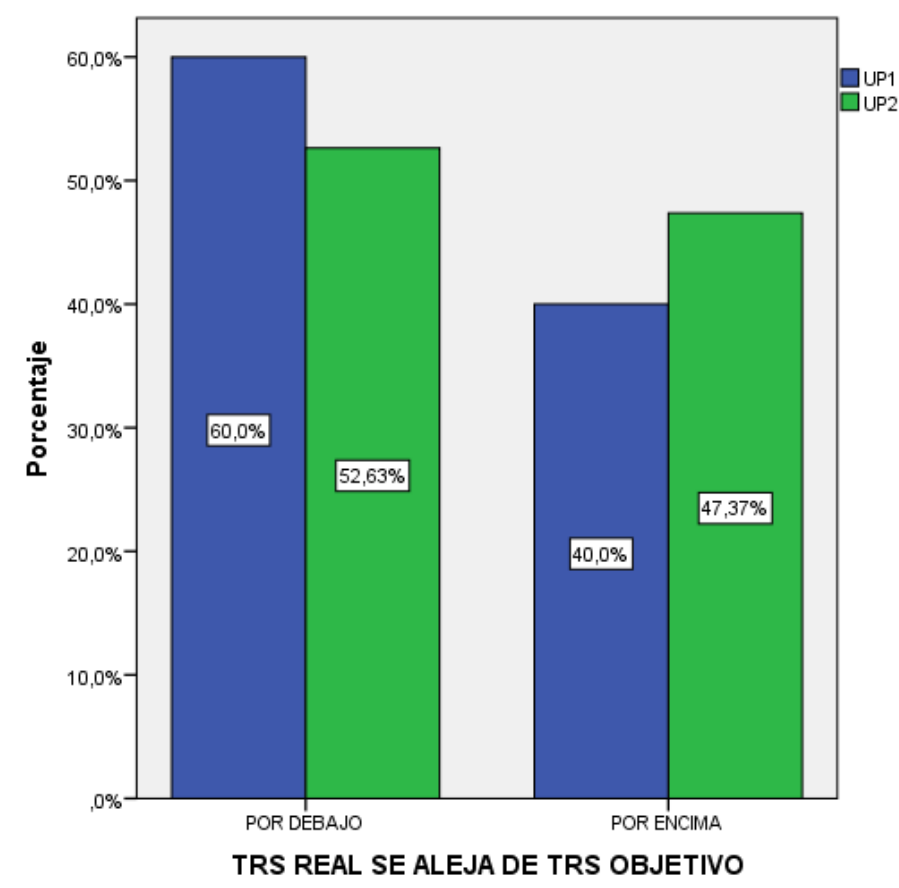

Fig. 2:Diagrama de barras. \% de líneas por debajo y por encima de la TRS objetivo en UP1 y UP2

El análisis descriptivo nos ha permitido identificar que existen diferencias entre las dos unidades de producción analizadas. Dichas diferencias son estadísticamente significativas en la proporción de líneas que se alejan de la TRS objetivo, así como en la TRS media, en el tiempo medio de pérdida total y en el tiempo medio de cambio cada UP 
(contraste de medias $\mathrm{T}$ con p-valor $<0,05$, es decir, $5 \%$ de error de tipo I), siendo más significativa en el turno de noche que en los de mañana o tarde.

En este sentido, es asimismo interesante destacar cómo, en comparación con la UP1, las líneas de la UP2 presentan unas tasas de rendimiento real con un componente laboral, según sea turno de mañana, tarde o noche, menos pronunciado. Concretamente, el 83,3\% de las líneas en la UP2 experimentan una tasa de rendimiento real más elevada en el turno de mañana si las líneas están activas dos turnos (mañana-tarde, mañana-noche, tarde-noche), manteniéndose la misma más o menos estable hasta el turno de noche, cuando la línea está activa durante los tres turnos. En cambio, las líneas de la UP1 presentan un patrón de actividad horaria claramente diferenciado a la UP2. La fluctuación de sus tasas de rendimiento por turno es el efecto de una actividad más baja a medida que pasa el día, cuando las líneas están activas durante los tres turnos $(36,6 \%) \mathrm{y}$, especialmente, en aquellas líneas de productos capilares. Se interpreta que las tasas de rendimiento con tres turnos reflejan aquellos periodos vitales en los que hay que trabajar más intensamente (alguna campaña). En las líneas de la UP1 que están activas dos turnos las tasas de rendimiento real son mayores en la mañana (o tarde) descendiendo en el turno de tarde (o noche), excepto en aquellas líneas dedicadas a tratamientos especiales y, por tanto, con menor demanda, en donde ocurre lo contrario.

También las diferencias son más que destacables entre las dos unidades de producción en cuanto al tiempo de cambio por formato, referencia o fórmula. Las dos unidades mantienen perfiles similares en dicha variable en cuanto al turno en que se produce. En el $72 \%$ de las líneas de UP1 el tiempo de pérdida se debe en mayor medida al tiempo de cambio por referencia en el turno de mañana, proporción que desciende en los turnos de tarde y noche. Siendo en UP2 cercano a $51 \%$. Aún son más destacables los porcentajes para el tiempo de cambio por jugo, entre el $75 \%$ y el $45 \%$ de las líneas que respectivamente conforman las dos unidades de producción. Finalmente, el tiempo de cambio por jugo es claramente menor en la UP1, sin que se pueda observar un patrón por turnos significativo.

Prácticamente todas las líneas son homogéneas en cuanto al tiempo de espera, averías y microparos, incluso por turnos.

\subsection{ANÁLISIS EXPLICATIVO-PREDICTIVO}

\subsubsection{Análisis discriminante}

Se emplea el análisis discriminante como técnica de dependencia, en la que se pretende averiguar si la pertenencia de las líneas a las dos unidades de producción preexistentes (variable dependiente) responde a causas objetivas, medidas a través de las variables métricas analizadas (variables independientes tiempo de pérdida, tiempo de ocupación, turno laboral, tiempo por limpieza y mantenimiento), o si por el contrario tales variables no permiten explicar la agrupación de las observaciones. Para evitar la duplicidad de información en el modelo se considera el tiempo de pérdida como única variable resumen, es decir, como suma de tiempo de averías, tiempo de esperas, microparos y tiempo de cambio de referencia, jugo o formato. No obstante, se han realizado otros análisis discriminantes teniendo en cuenta la información de estas últimas variables y no el tiempo de pérdida total, pero la capacidad predictiva era inferior para estos casos con el prácticamente mismo poder discriminante cada una de ellas.

La ecuación lineal discriminante, similar a la de regresión lineal múltiple, es la siguiente:

$$
\begin{aligned}
& \text { Pertenencia al grupo i (unidad de producción) }=B_{0}+B_{1} X_{1}+B_{2} X_{2}+\ldots+B_{p} X_{p} \\
& \qquad(i=1,2)
\end{aligned}
$$

donde:

$\mathrm{X}$ : son las variables independientes

$\mathrm{B}_{0}$ : es la constante

$\mathrm{B}_{\mathrm{p}}$ : son los coeficientes estimados a partir de los datos originales de modo que los valores de la función difieran el máximo posible entre los grupos, de acuerdo a la Tabla 3. 


\begin{tabular}{|l|c|c|}
\cline { 2 - 3 } \multicolumn{1}{c|}{} & \multicolumn{2}{c|}{$\begin{array}{c}\text { Coeficientes estimados B para cada } \\
\text { Unidad de Producción }\end{array}$} \\
\cline { 2 - 3 } & UP1 & UP2 \\
\hline Tiempo de pérdida & $8,059 * * *$ & 7,196 \\
Tiempo de ocupación &,$- 784^{*}$ &,- 929 \\
Turno laboral &,$- 611^{*}$ &,- 909 \\
Tiempo de limpieza &, 320 &, 276 \\
Tiempo por mantenimiento & 2,526 & 2,703 \\
(Constante) & $-112,631 * *$ & $-107,871$ \\
\hline
\end{tabular}

Nota: los coeficientes estadísticamente significativos están especificados con $* * *$ p-valor $<0,01 ; * *$ p-valor $<0,05 ; *$ p-valor $<0,1$

Tabla 3: Coeficientes de la función de clasificación. Funciones discriminantes lineales de Fisher

La diferenciación significativa en las líneas en las dos unidades de producción se debe fundamentalmente al tiempo de pérdida, variable con mayor poder discriminante ( $\mathrm{p}$-valor $<0,01)$. Además, existen diferencias, aunque no tan significativas, en el tiempo de ocupación y en el turno laboral. Las puntuaciones medias en el tiempo de limpieza y por mantenimiento (mecánicos) son iguales con un nivel de confianza del 95\%, por lo que aquí no existirían diferencias significativas entre unidades de producción ( $\mathrm{p}$-valor $>0,05$ ). La capacidad explicativa del análisis estadístico llevado a cabo es representativa en términos generales, como se muestra en la Tabla 4, pues clasifica correctamente el $82,1 \%$ de las líneas.

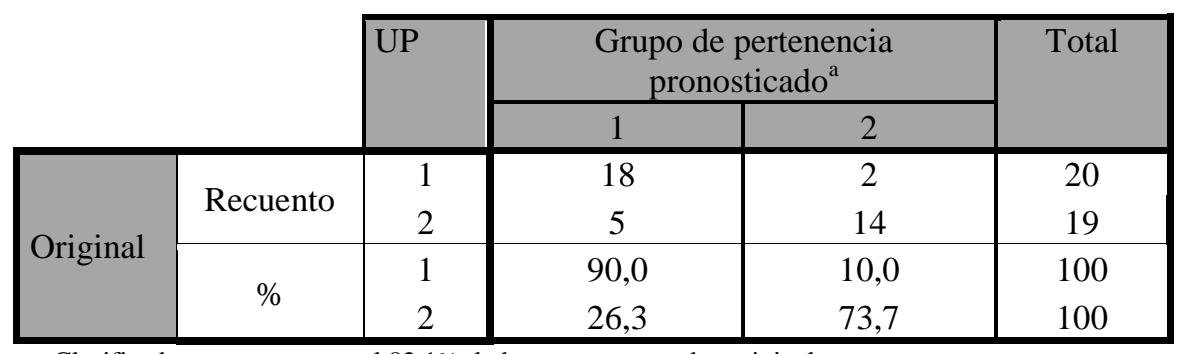

a. Clasificados correctamente el $82,1 \%$ de los casos agrupados originales

\section{Tabla 4: Resultados de la clasificación en el análisis discriminante}

\subsubsection{Análisis de regresión lineal}

Con la regresión, aparte de medir el grado de asociación entre las variables consideradas, se pueden realizar predicciones de la variable dependiente. Es decir, se establecen relaciones entre variables que se presupone que tienen relación con el objeto de estudio (diferencia entre la TRS real y la objetivo) y, consecuentemente, prever su comportamiento futuro, incluso si las condiciones varían.

El análisis de regresión lineal ha llevado asociado una serie de estrategias de diagnóstico (análisis de los residuos, análisis de no colinealidad) que informan de su estabilidad e idoneidad en el cumplimiento de las condiciones básicas necesarias para garantizar la validez del modelo. El método de selección de variables en el análisis de regresión lineal fue eliminación hacia atrás.

El modelo de regresión lineal múltiple (MRLM) puede ser formulado de manera simplificada como se muestra en la ecuación 2:

donde

$$
\mathrm{Y}_{\mathrm{i}}=\beta_{0}+\beta_{1} \mathrm{X}_{\mathrm{i} 1}+\beta_{2} \mathrm{X}_{\mathrm{i} 2}+\beta_{3} \mathrm{X}_{\mathrm{i} 3}+\ldots+\beta_{\mathrm{p}-1} \mathrm{X}_{\mathrm{i}, \mathrm{p}-1}+\varepsilon_{\mathrm{i}}
$$


$\mathrm{Y}_{\mathrm{i}}=$ variable dependiente

$\mathrm{X}_{\mathrm{i}}=$ variables Independientes

$\beta=$ coeficientes desconocidos

$\varepsilon_{\mathrm{i}}=$ término de error; $\mathrm{i}=1,2, . . \mathrm{n}$

Los resultados detallados del modelo, que se explican a continuación, se muestran en la Tabla 5.

\begin{tabular}{|c|c|c|c|}
\hline \multirow[t]{2}{*}{ Modelo para UP1 } & \multicolumn{2}{|c|}{ Coeficientes estimados } & \multirow{2}{*}{$\mathbf{R}^{2}$ corregido } \\
\hline & $\beta$ & p-valor & \\
\hline $\begin{array}{ll} & \text { (Constante) } \\
1 & \text { TIEMPO AVERIAS } \\
& \text { TIEMPO CAMBIO } \\
2 & \text { (Constante) } \\
2 & \text { TIEMPO CAMBIO }\end{array}$ & $\begin{array}{r}-9,334 \\
, 023 * * \\
, 301 * * \\
-10,294 \\
\mathbf{1 , 0 3 2} * *\end{array}$ & $\begin{array}{l}, 255 \\
, 030 \\
, 038 \\
, 178 \\
\mathbf{, 0 2 0}\end{array}$ & $\begin{array}{l}, 451 \\
, 707\end{array}$ \\
\hline \multirow[t]{2}{*}{ Modelo para UP2 } & \multicolumn{2}{|c|}{ Coeficientes } & \multirow{2}{*}{$\mathbf{R}^{2}$ corregido } \\
\hline & $\beta$ & p-valor. & \\
\hline $\begin{array}{ll} & \text { (Constante) } \\
1 & \text { TIEMPO AVERIAS } \\
& \text { TIEMPO CAMBIO } \\
2 & \text { (Constante) } \\
& \text { TIEMPO CAMBIO } \\
\end{array}$ & $\begin{array}{l}-8,278 \\
, 334 * * \\
, 441 * * \\
-5,924 \\
, \mathbf{5 7 8} * * *\end{array}$ & $\begin{array}{l}, 096 \\
, 033 \\
, 034 \\
, 118 \\
\mathbf{, 0 0 2} \\
\end{array}$ & $\begin{array}{l}, 567 \\
, 713 \\
\end{array}$ \\
\hline \multirow[t]{2}{*}{ Modelo conjunto } & \multicolumn{2}{|c|}{ Coeficientes } & \multirow{2}{*}{$\mathbf{R}^{2}$ corregido } \\
\hline & $\beta$ & p-valor & \\
\hline $\begin{array}{ll} & \text { (Constante) } \\
\text { TIEMPO CAMBIO }\end{array}$ & $\begin{array}{l}-2,334 * \\
, 301 * * * \\
\end{array}$ & $\begin{array}{l}, 095 \\
, 008\end{array}$ & ,782 \\
\hline
\end{tabular}

Nota: los coeficientes estadísticamente significativos están especificados con

$* * *$ p-valor $<0,01 ; * *$ p-valor $<0,05 ; *$ p-valor $<0,1$

Tabla 5:Modelos de regresión para la variable dependiente diferencia entre la TRS real y la objetivo

Una variable tiene más peso (importancia) en el modelo de regresión cuanto mayor es su coeficiente $\beta$ en valor absoluto. Esto significa que esta variable es un buen predictor de la variable dependiente. Inicialmente se elaboraron 5 modelos de regresión lineal múltiple utilizando las variables tiempo de averías, microparos, esperas y tiempo de cambio. El mejor modelo explicativo de la diferencia entre la TRS real y la TRS objetivo muestra únicamente dos variables estadísticamente significativas, tiempo de averías y tiempo de cambio (p-valor < 0,05). Sin embargo, en el modelo explicativo con las dos variables independientes (con coeficientes $\beta$ iguales a 0,023 y 0,301 para la UP1 y 0,334 y 0,441 para la UP2) el $\mathrm{R}^{2}$ corregido es más bajo que considerando sólo el tiempo de cambio (poder explicativo del $45,1 \%$ y $56,7 \%$, respectivamente, frente a $70,7 \%$ y $71,3 \%$ ). Por tanto, suponer que estos dos factores son determinantes en la diferencia entre la TRS real y la TRS objetivo no sería del todo correcto. Además, si consideramos conjuntamente las dos UP también la variable independiente con mayor poder explicativo es el tiempo de cambio (p-valor $<0,01)$. El grado de ajuste que explica hasta qué punto el modelo es capaz de predecir la diferencia entre la TRS real y la objetivo es relativamente aceptable de acuerdo a la Tabla $5\left(\mathrm{R}^{2}\right.$ corregido $\left.=78,2 \%\right)$.

\subsubsection{Análisis de regresión logística}

Como la TRS es un indicador del rendimiento global, se ha considerado relevante indagar los factores explicativos de la diferencia de la TRS objetivo y real. Dicha variable es de carácter binario distinguiéndose la condición de estar por 
debajo o por encima de la TRS objetivo prefijada por línea. De ahí la utilización del modelo de respuesta cualitativa regresión logística.

Esta metodología permite no sólo calcular, en escala logarítmica, la probabilidad de que una línea ya observada pertenezca al grupo de los que están por debajo del objetivo prefijado por línea, sino también clasificar una línea cualquiera (de la que se quiere saber si va a conseguir la tasa objetivo o no) de acuerdo a una serie de variables explicativas clave. Así, a través del análisis de regresión logística binaria, se ha examinado si el turno (mañana, tarde o noche), el tiempo de ocupación, el tiempo de pérdida por avería, por microparos, por esperas o por cambio de formato, jugo o referencia pueden explicar la diferencia (positiva o negativa) entre la TRS real y la TRS objetivo por línea. El análisis revela que las variables tiempo de pérdida por avería y por cambio de formato, jugo o referencia, más que el turno laboral, tiempo de ocupación o el tiempo de pérdida por microparos o esperas, determinan la probabilidad de alejarse de la TRS objetivo por línea.

La presencia de la interacción entre variables independientes puede alterar la interpretación de los resultados del modelo porque el efecto de una variable independiente cambia en función del nivel de una segunda variable independiente (el "sobreaumento" de la explicación de la variable dependiente). Así, para controlar el efecto conjunto de los tiempos alcanzados de acuerdo con el turno laboral, se han incluido las interacciones del turno en que se producen los tiempos de pérdida u ocupación. Sólo el efecto combinado del tiempo pérdida por microparos con el turno laboral contribuye al poder explicativo del modelo.

La metodología adoptada permite estimar la probabilidad de alejarse de la TRS objetivo de cualquier línea de acuerdo a estas variables explicativas clave. El modelo obtenido proporciona un porcentaje elevado de casos de la muestra correctamente clasificados $(84,6 \%)$. Por tanto, es de esperar que dicho modelo muestre buenos resultados a la hora de predecir la condición de estar por encima o por debajo de la TRS objetivo para cualquier línea.

Además, la calidad del potencial explicativo del análisis se refleja a través de $\mathrm{R}^{2}$ de Nagelkerke $(0,649)$. La interpretación de este indicador es análoga a la del $\mathrm{R}^{2}$ en los modelos lineales tradicionales explicando el modelo resultante casi el $65 \%$ de la variabilidad total de la variable dependiente.

El modelo parte de la expresión de la probabilidad de un suceso, $\mathrm{p}_{\mathrm{i}}$, de acuerdo a la ecuación (3):

$$
p_{i}=\frac{1}{1+e^{-\beta_{0}-\sum_{i=1}^{n} \beta_{i} x_{i}}}
$$

donde las $\mathrm{x}_{\mathrm{i}}$ son las distintas variables explicativas. En nuestro caso será la estimación de que una línea se aleje por debajo de la TRS objetivo. Del mismo modo 1- $\mathrm{p}_{\mathrm{i}}$ será la estimación de que una línea se aleje por encima de la TRS objetivo, es decir, de acuerdo a la ecución (4)

$$
1-p_{i}=\frac{e^{-\beta_{0}-\sum_{i=1}^{n} \beta_{i} x_{i}}}{1+e^{-\beta_{0}-\sum_{i=1}^{n} \beta_{i} x_{i}}}=\frac{1}{1+e^{\beta_{0}+\sum_{i=1}^{n} \beta_{i} x_{i}}}
$$

El cociente $\frac{p_{i}}{1-p_{i}}=o d d s$ representa las veces que es más probable que la línea esté por debajo que de que lo esté por encima. Y resulta que $g_{i}=\lg \frac{p_{i}}{1-p_{i}}=\beta_{0}+\sum_{i=1}^{n} \beta_{i} x_{i}$ es el modelo que recibe el nombre de logit y que permite explicar en escala logarítmica la probabilidad de pertenecer a uno u otro grupo.

Los coeficientes de regresión $B$ indican la influencia relativa de cada variable predictora sobre la variable dicotómica. De forma intuitiva, un coeficiente positivo aumenta la probabilidad, mientras que un valor negativo disminuye la probabilidad predicha. Las $\operatorname{Exp}(\beta)$ u odd ratio se interpretan como el cambio en la probabilidad de estar por debajo de la TRS objetivo (categoría escogida como referencia de la variable dependiente y), asociado a cada una de las categorías de las variables independientes o explicativas (x) en relación a la categoría de referencia, en nuestro caso estar por 
debajo de la TRS objetivo. De acuerdo a la Tabla 6, la probabilidad de estar por debajo de la TRS objetivo cuando el turno laboral es tarde o noche existiendo tiempo de pérdida por microparos sería aproximadamente 1,8 y 1,1 veces superior, respectivamente, a la situación que se de turno de mañana.

En términos generales, en las variables independientes, una odd ratio $>1$ indica una probabilidad mayor de estar por debajo de la TRS objetivo, mientras que una odd ratio <1 representa una mayor probabilidad de estar por encima de la TRS objetivo.

\begin{tabular}{|c|c|c|c|}
\hline & $\begin{array}{c}\text { B } \\
\text { Coeficientes estimados }\end{array}$ & p-valor & $\operatorname{Exp}(B)$ \\
\hline Turno & \multicolumn{3}{|c|}{, 316} \\
\hline \multicolumn{4}{|l|}{ Mañana (ref.) } \\
\hline Tarde (1) & ,456 & ,232 & 1,578 \\
\hline Noche (2) & ,367 &, 214 & 1,443 \\
\hline Tiempo ocupación &,- 340 & 0,321 & 0,712 \\
\hline \multicolumn{4}{|l|}{ Tiempo pérdida } \\
\hline Tiempo pérdida avería &, $766 * *$ &, 013 & 2,151 \\
\hline Tiempo pérdida microparos & ,494 &, 713 & 1,639 \\
\hline Tiempo pérdida cambio formato, jugo o referencia &, $542 * *$ &, 011 & 1,719 \\
\hline \multicolumn{4}{|l|}{ Interacciones } \\
\hline Tiempo ocupación * turno & &, 163 & \\
\hline \multicolumn{4}{|l|}{ Tiempo ocupación $*$ turno mañana } \\
\hline Tiempo ocupación $*$ turno tarde & $-2,313$ &, 214 & ,099 \\
\hline Tiempo ocupación * turno noche & $-1,567$ & ,232 & ,209 \\
\hline Tiempo pérdida averías* turno & & ,218 & \\
\hline \multicolumn{4}{|l|}{ Tiempo pérdida averías* turno mañana } \\
\hline Tiempo pérdida averías* turno tarde & $-1,234$ &, 125 &, 291 \\
\hline Tiempo pérdida averías* turno noche & $-2,433$ &, 142 & ,088 \\
\hline Tiempo pérdida microparos* turno & & ,000 & \\
\hline \multicolumn{4}{|l|}{ Tiempo pérdida microparos* turno mañana } \\
\hline Tiempo pérdida microparos $*$ turno tarde &, $634 * * *$ &, 002 & 1,885 \\
\hline Tiempo pérdida microparos* turno noche &, $064 * * *$ &, 003 & 1,066 \\
\hline Tiempo pérdida cambio* turno & & ,203 & \\
\hline \multicolumn{4}{|l|}{ Tiempo pérdida cambio* turno mañana } \\
\hline Tiempo pérdida cambio* turno tarde & 2,345 &, 212 & 1,409 \\
\hline Tiempo pérdida cambio* turno noche & 0,343 &, 107 &, 118 \\
\hline Constante & $-2,141^{*}$ &, 095 & ,086 \\
\hline
\end{tabular}

Nota: las odds ratio estadísticamente significativas están especificadas con

$* * *$ p-valor $<0,01 ; * *$ p-valor $<0,05 ; *$ p-valor $<0,1$

Tabla 6: Modelo de Regresión logística sobre la condición de estar por debajo o por encima de la TRS objetivo

Se considera que la variable es estadísticamente significativa si lleva asociado un p-valor $<0,05$. Las conclusiones sobre los factores determinantes de la probabilidad de alejarse por debajo o no de la TRS objetivo por línea, son las siguientes:

1. Las variables explicativas más importantes se asocian al tiempo de pérdida, siendo la más significativa el tiempo de pérdida por cambio de referencia, jugo o formato. 
2. Sin embargo, el efecto combinado del tiempo de pérdida por microparos con el turno laboral en el que se produce (no cada variable por separado) sí contribuye al poder explicativo del modelo.

3. El tiempo de ocupación no parece agregar poder explicativo al modelo en su conjunto, ni en su interacción con el turno laboral.

4. La variable tiempo de pérdida por averías es significativamente considerable en el modelo, aumentando la probabilidad de estar por debajo de la TRS objetivo 2,15 veces a medida que se incrementa el valor de dicha variable. Esto significa que a más tiempo de pérdida por avería, mayor es la probabilidad de estar por debajo de la TRS objetivo.

\section{DISCUSIÓN Y CONCLUSIONES}

Este trabajo de investigación aplica el análisis estadístico a la información proporcionada por una empresa del sector cosmético, no solo de una manera descriptiva, sino explicativa y predictiva. Hoy en día, la mayoría de las empresas se enfrentan a grandes cantidades de información y datos careciendo, en muchos casos, de la capacidad de aprovecharlos como ventaja competitiva en su toma de decisiones [22]. En este sentido, no hemos encontrado ningún trabajo que examine los factores explicativos de la tasa de rendimiento en un intento de mejora del indicador OEE dentro de "programas de aprendizaje". Este trabajo se desarrolla con el objetivo de identificar dichos factores y proponer de acuerdo a los resultados empresariales una serie de recomendaciones en su plan de mejora.

En cuanto a los análisis estadísticos llevados a cabo, los resultados se pueden sintetizar en las siguientes conclusiones:

1. Las dos unidades de producción se diferencian significativamente en el indicador de rendimiento TRS.

2. Una vez diagnosticado que la variable con mayor poder discriminante en la diferenciación en grupos es el tiempo de pérdida, el modelo de regresión lineal múltiple señala que los factores de tiempo de cambio son los que más inciden en la diferencia dela TRS real y la objetivo, siendo la variable tiempo de cambio por formato la de mayor poder explicativo dentro de estos factores.

3. Las variables determinantes que significativamente permiten diferenciar a los grupos de líneas que se alejan de la TRS objetivo en cada unidad de producción, son tiempo de pérdida por avería y por cambio de formato, jugo o referencia, más que el turno laboral, tiempo de ocupación o el tiempo de pérdida por esperas o microparos. Aunque paralelamente se aprecia su influencia desfavorable cuando se interacciona esta última variable con el turno donde se produce.

Tras el análisis de estos resultados empíricos, se podría establecer un plan de mejora en la empresa consistente en las siguientes recomendaciones como contribución a nivel práctico del trabajo presentado:

1. Revisar la manera en que se están realizando los cambios de formato, formando equipos con los trabajadores involucrados en estas líneas y pidiéndoles propuestas de realización de dichos cambios. Medir el rendimiento una vez implantados los cambios y cuantificar la mejora en el cálculo del indicador OEE. El valor del OEE permitirá clasificar una o más líneas de producción, o toda una planta, con respecto a las mejores de su clase y que ya han alcanzado el nivel de excelencia.

2. Implantar un sistema SMED en aquellas líneas con mayores tiempos de espera en los cambios de formato.

3. Revisar en cada uno de los turnos los tiempos de pérdida por microparos más significativos, buscándoles explicación, siempre con ayuda de los propios operarios.

4. Revisar el plan de mantenimiento de la empresa, proponiendo un Plan de Mantenimiento Productivo Total para reducir los tiempos de paradas por averías.

En definitiva, como aspecto aportador del artículo está el análisis de "los mudas" o tiempos no productivos que nos lleva a un rediseño de procesos, que afectaría a la TRS y, por tanto, al cálculo del indicador OEE. Una TRS alta es relativamente bueno, pues implica que nuestro equipamiento está trabajando a un buen rendimiento global; no obstante, también puede implicar una señal de alarma, pues el equipamiento actual no podrá absorber una subida repentina de las necesidades de producción necesitándose nueva maquinaria. Habrá que evaluar cuál es el punto adecuado según la situación particular. 
Lo mejor y más práctico es que el cálculo del indicador OEE sea llevado a cabo y controlado por los propios operadores del proceso, es decir, existe la necesidad de tomar métricas en el lugar donde ocurren los hechos. Para esta tarea es fundamental contar con un grupo de trabajo multidisciplinar en el que se puedan resolver las situaciones planteadas para las diferentes causas raíz de cada una de las pérdidas, priorizando siempre aquellas de mayor impacto en tiempos, coste o facilidad de ejecución. Algunas de las ventajas de abordar la mejora del OEE en una instalación o proceso es que se obtienen resultados en el corto plazo y fundamentalmente nos permite una elevada motivación del personal. Se logra involucrarle mediante formación y entrenamiento, para que con su participación activa, consiga los resultados, emplee las herramientas asociadas y sea capaz de aplicarlas con autonomía y de una manera constante y sistemática.

Así, y de acuerdo a nuestra hipótesis de investigación, los operarios serán los más afectados por esta metodología de medición por lo que es vital que ellos mismos hayan sido formados en los conceptos y en la forma de cálculo y sean ellos quienes lleven adelante la medición, el control y la planificación de la mejora en ese "proceso de aprendizaje". De esta manera se refuerza el sentimiento de propiedad sobre los equipos a los que les dedican su atención durante el turno de operación. Es muy recomendable acompañar este tipo de abordajes con Sistemas de Sugerencias. De esta forma se gestiona el cambio cultural que es requerido para la Mejora Continua.

Cabe hacer énfasis también en que abordando la mejora del OEE afloran fuentes de ineficiencia que, con otro sistema de medida, hubieran quedado ocultas. A tal efecto se hace indispensable la utilización de gestión visual colocada en un lugar bien accesible para quienes aplican esa información de manera que permita a todos monitorear los avances y ver las tendencias.

El estudio realizado no está exento de limitaciones. Entre ellas la conveniencia de extender el estudio a otras fábricas del grupo con el objetivo de garantizar la generalización de los resultados obtenidos, e incluso a otros sectores. Así como ampliar el "programa de aprendizaje" al otro bloque de pérdidas que forma parte de la métrica del OEE: calidad. Además, la verdadera clave del éxito se encuentra en el nivel de convicción que tiene la alta dirección sobre este tema y la capacidad de transmitir a sus gerentes la importancia de dedicar tiempo de su agenda y de su gente a un tema tan relevante para la empresa. Creemos que el conjunto de actividades de éxito para lograr una mejora del indicador OEE se inicia con la creación de un entorno empresarial que promueve la calidad, seguido de una comprensión de sus principios y, finalmente, de un esfuerzo por involucrar a los empleados en las actividades necesarias para su consecución. Si se hacen bien todas estas cosas, la organización normalmente satisfará a sus consumidores y conseguirá una ventaja competitiva.

Como parte del debate sobre el tema, a partir de la propuesta presentada en el artículo, se plantea el desarrollo y aplicación práctica de las diferentes propuestas de mejora con objeto de verificar el grado y la magnitud de mejora aportados en cada unidad de producción estudiada, lo cual formaría parte de futuros trabajos de investigación.

Finalmente, a nivel académico consideramos que se necesitan más estudios de este tipo donde haya una transferencia de conocimientos activa entre las universidades o los centros de investigación y las empresas.

\section{AGRADECIMIENTOS}

Esta investigación está basada en la información proporcionada por la dirección de una fábrica del sector cosmético, a quien los autores agradecen su colaboración y ayuda en la recopilación y entrega de datos. No obstante, por cuestiones de confidencialidad no se proporcionan nombres. 


\section{BIBLIOGRAFÍA}

[1] Eguren-Egiguren J.A, Goti-Elordi A, Pozueta-Fernández L. "Diseño, aplicación y evaluación de un modelo para la mejora de procesos en sectores industriales maduros. Estudio del caso". DYNA Ingeniería e Industria. Febrero 2011. Vol. 86-1 p. 59-73.

[2] Imai, M, "Kaizen: la clave de la ventaja competitiva japonesa". CECSA Editorial, México, D.F., 1ª edición 1989. ISBN: 968-26$1128-8$

[3] Bozdogan K. "Towards an Integration of the Lean enterprise system, Total Quality Management, Six Sigma and related enterprise process improvement methods". Encyclopedia of Aerospace Engineering, John Wiley \& Sons, 2010. Chapter: Evolution of the Lean Enterprise System, in Section 5- Manufacturing and Lean Technologies.

[4] Bozdogan K. "Evolution of the Lean Enterprise System: a critical synthesis and agenda for the future". Encyclopedia of Aerospace Engineering, John Wiley \& Sons, 2010. Chapter: Evolution of the Lean Enterprise System, in Section 5- Manufacturing and Lean Technologies.

[5] Suárez-Barraza M.F, Castillo-Arias I, Miguel-Dávila J.A. "La aplicación del Kaizen en las organizaciones mexicanas. Un estudio empírico". REVISTA Globalización, Competitividad y Gobernabilidad. Febrero 2011. p. 60-74. DOI: http://dx.doi.org/10.3232/gcg.2011.V5.N1.04

[6] Suárez-Barraza M.F, Miguel-Dávila J.A. "Implementación del Kaizen en México: un estudio exploratorio de una aproximación gerencial japonesa en el contexto latinoamericano”. Innovar. Revista de Ciencias Administrativas y Sociales. Julio-Septiembre 2011. p. 19-37.

[7] De Domingo, J., Arranz, A. Calidad y Mejora Continua. Editorial Donostiarra, 2002. 350 pág. ISBN 9788470632143

[8] Jaca-García C, Santos-García J. "La Mejora Continua en las organizaciones. Análisis de su implantación en 30 empresas". DYNA. Marzo 2009. Vol.84-2 p.133-140. DOI: http://dx.doi.org/10.6036/1964

[9] García Morales, V.; Lloréns Montes, F.J.; Verdú Jover, A. "Antecedents and Consequences of Organizational Innovation and Organizational Learning in Entrepreneurship". Industrial Management \& Data Systems. 2006. Vol. 106 number. 1, p. 21-42. DOI: http://dx.doi.org/10.1108/02635570610642940

[10] Chakravorty, S. "Six Sigma programs: An implementation model". International Journal Production Economics. 2009. Vol. 119 p.1-16. DOI: http://dx.doi.org/10.1016/j.ijpe.2009.01.003

[11] Bendell, T. "A review and comparison of Six Sigma and the lean organizations". The TQM Magazine. 2006. Vol.18 Issue 3 p.255-262. DOI: http://dx.doi.org/10.1108/09544780610659989

[12] Womack J.P, Jones D.T., Roos D. The Machine that changed the World, The Story of Lean Production, HarperPerennial, 1991. pág. 323. ISBN- 10: 0060974176 / ISBN- 13: 9780060974176

[13] Womack J.P, Jones D.T. Lean Thinking. Gestión 2000, 2012. p. 504. ISBN 9788498750218

[14] Chen L, Meng B. "The Application of Setup Reduction in Lean Production”. Asian Social Science. July 2010 Vol. 6, N7.

[15] Ohno T. Toyota Production System, $1^{\text {st }}$ edition (March 1, 1988) June 1988, Productivity Press, p. 152. ISBN-10: 0915299143 ISBN-13: 978-0915299140

[16] Sanchís R., Poler R, Mula J., Peidro, D. “Gestión de la calidad total y mantenimiento productivo total en la fabricación de alto rendimiento". DYNA. Diciembre 2011.Vol. 86-6 p. 648-655. DOI: http://dx.doi.org/10.6036/4106

[17] Mansour H., Ahmad M., Ahmed H. "Potential using of OEE in evaluating the operational performance of workover activities". Advances in Sustainable and Competitive Manufacturing Systems. 23rd International Conference on Flexible Automation \& Intelligent Manufacturing. Springer. 2013. p. 877-886. DOI: http://dx.doi.org/10.1007/978-3-319-00557-7_72

[18] Garza-Reyes J.A., Eldridge S., Barber K.D. et al. "Overall equipment effectiveness (OEE) and process capability (PC) measures A relationship analysis". International Journal of Quality \& Reliability Management. 2010. Vol. 27 No. 1. p. 48-62. DOI: http://dx.doi.org/10.1108/02656711011009308

[19] Ahuja I.P.S., Khamba J.S. "An evaluation of TPM initiatives in Indian industry for enhanced manufacturing performance". International Journal of Quality \& Reliability Management. $2008 . \quad$ Vol. 25 Issue 2 p.147-172. DOI: http://dx.doi.org/10.1108/02656710810846925

[20] Makrymichalos, M., Antony, J., Antony, F. et al. "Statistical thinking and its role for industrial engineers and managers in the 21st century". Managerial Auditing Journal. 2005. Vol. 20 Issue 4, p. 354- 363. DOI: http://dx.doi.org/10.1108/02686900510592043 [21] Kendall M. Multivariate Analysis. $2^{\text {nd }}$ edition. London: McMillan, 1980. pág 212. Griffin's Statistical Monographs and Courses, $\mathrm{N}^{\circ}$ 2. ISBN: 0028477901, 9780028477909

[22] Hoerl, R.W., Snee, R.D. Statistical Thinking: Improving Business Performance. Duxbury Press, Belmont, CA, 2002. 